\title{
Planificación académica y cultura organizacional en las instituciones de educación básica
}

\section{Academic Planning and Organizational Culture in the Institutions of Basic Education}

\author{
Juana Ojeda \\ Universidad Nacional Abierta y Universidad del Zulia \\ Venezuela \\ juanacecilia@hotmail.com \\ María Ferrer \\ Universidad Pedagógica Experimental Libertador \\ Venezuela \\ marchiferrer@hotmail.com
}

Recibido 06-07-2010 • Aceptado 06-09-2010 • Corregido 24-10-2010

Resumen: En este artículo se hace un resumen de un estudio que tuvo como propósito determinar la relación entre la cultura organizacional y la planificación académica en las instituciones de educación básica. El clima organizacional estuvo evaluado a partir de los elementos, categorías y tipos que lo modifican; por su parte, la planificación académica se midió a partir de las fases, principios y pasos previstos en sus objetivos. Para alcanzar el propósito establecido, se hizo una exhaustiva revisión bibliográfica que permitió fundamentar teóricamente la investigación. La construcción teórica-referencial se hizo por medio de la revisión de antecedentes, análisis de teorías, definición de conceptos y fundamentos teóricos relacionados con la temática que se investigó. El tipo de investigación utilizada fue la descriptiva. La información se obtuvo mediante la aplicación de un cuestionario aplicado al personal docente y directivo de las instituciones de educación básica investigadas. El análisis y la interpretación de los resultados obtenidos se efectuaron de manera cuantitativa utilizando el paquete estadístico computarizado denominado SPSS versión 12.0. Se logró determinar que existe una alta relación de dependencia entre las variables

\section{Introducción}

Hoy se hace necesario promover una verdadera innovación educativa que converja con la situación por la cual atraviesa Venezuela y que ejerza una acción más real en la transformación del funcionamiento de la sociedad y su medio. Frente a este escenario se deben dirigir las políticas educativas hacia las instituciones escolares sobre las cuales recae la responsabilidad de ejecutar y cumplir sus objetivos. Para lograr estos fines las instituciones educativas requieren de una planificación académica que esté en concordancia con su cultura organizacional, de manera tal que provea los elementos, categorías y tipos de cultura que deben tomarse en cuenta durante este proceso.

En este contexto, cobra relevancia la planificación académica como elemento 
clima organizacional y planificación académica, lo que puso en evidencia que la planificación académica es afectada por los elementos, categorías y tipos de clima organizacional imperantes en las instituciones básicas estudiadas.

Palabras clave: Clima, planificación, cultura, educación.

Abstract: This study was intended to determine the relationship between organizational culture and academic planning in basic education institutions. Organizational climate was evaluated by elements, categories and types of amending, whereas academic planning was measured on stages, principles and steps provided for its objectives. To achieve the established purpose, an exhaustive literature review was formed that allowed theoretically based research. Themes that were investigated included the theoretical-referential construction on revision history, analysis of theories, definition of concepts and related theoretical foundations. The type of research used was descriptive. Information was obtained through a questionnaire applied to the teaching and managerial staff of the basic education institutions under investigation. The analysis and interpretation of the results achieved were made in a quantitative manner through a computerized statistical package called SPSS version 12.0. It was able to determine that there is a high dependency ratio between organizational climate variables and academic planning, proving that academic planning is affected by elements, categories and types of organizational climate rulings in the basic institutions studied.

Key words: Education, culture, climate, planning. fundamental y necesario para guiar $\mathrm{y}$ promover el desarrollo de los objetivos institucionales establecidos en el currículo fomentando un ambiente de apertura que permita a todos los miembros de la comunidad educativa su participación en la toma de decisiones y la discusión que favorezca el desarrollo integral del educando como ser integrador de la sociedad.

A pesar de la relevancia que tiene la planificación académica y la cultura organizacional en las escuelas, la realidad presenta una serie de errores que inciden en un funcionamiento deficiente y que afecta la labor del personal docente debido a la falta de conocimientos en la misma, entre los que se destacan: una carencia de planificación de la enseñanza que permita garantizar el cumplimiento de la función docente, una toma de decisiones que contradice los patrones culturales (artefactos, valores adoptados y supuestos básicos) que caracterizan las instituciones educativas y un exceso de lineamientos tradicionales directamente relacionados con la comunidad local, los padres de familia, el personal directivo y los docentes.

Lo anterior se aleja de una concepción de la educación dentro del ámbito social como un instrumento de desarrollo que atribuye a la instrucción escolar formal las funciones de transmisión de cultura de generación en generación y la formación de integral de sus educandos de manera sistemática dentro de la cultura imperante, en la comunidad educativa y en la sociedad.

Desde este punto de vista, se consideran de primordial importancia la planificación académica y la cultura organizacional en la actividad educativa, especialmente en los niveles de educación básica, donde los resultados constituyen insumos que contribuyen al mejoramiento de la calidad de los procesos educativos.

La actividad de educación y formación del talento humano se ha convertido en los últimos años en uno de los principales motores del desarrollo cultural, político, social y económico en los países 
latinoamericanos. No obstante, numerosos países en desarrollo se encuentran desprovistos de estos servicios y sufren un déficit de conocimientos, lo cual se opone a la efectividad en sus sistemas educativos, hecho que está marcado, según Gómez (2003), en la falta de planificación de estrategias instruccionales de enseñanza que contribuyan al mejoramiento cualitativo del estudiante como ser integral.

La educación venezolana define los componentes curriculares y su finalidad para construir un modelo orientado por las políticas educativas y las determinaciones contextuales, las cuales deben dar respuesta a las exigencias de formación del educando en todos sus niveles educativos; estas exigencias están orientadas a flexibilizar y orientar la práctica del personal docente en su rol de planificador del proceso de enseñanza-aprendizaje y así ampliar las posibilidades de integración e interacción entre los sujetos vinculados con la organización escolar.

Es pertinente utilizar el concepto de planificación para hacer referencia al proceso de formulación y definición de objetivos y prioridades a nivel macrosocial; o bien, en relación con una rama o sector (económico, educativo, social). La planificación alude a un proceso de tipo global, que se formula en función de satisfacer las necesidades sociales, por medio de la realización de un plan. Para Ander-Egg (1996) la planificación como la acción consistente en utilizar un conjunto de procedimientos mediante los cuales se introduce una mayor racionalidad y organización en un conjunto de actividades $y$ acciones articuladas entre sí que, previstas anticipadamente, tienen el propósito de influir en el curso de determinados acontecimientos, con el fin de alcanzar una situación elegida como deseable mediante el uso eficiente de medios escasos o limitados.

Actualmente, en la planificación no se excluyen las tareas de programación a nivel global por ser necesarias para articular el funcionamiento del sistema educativo en su conjunto; sin embargo, la planificación académica se realiza casi exclusivamente a nivel microsocial, con el propósito de programar las actividades y elaborar el proyecto educativo y curricular de las instituciones educativas.

En la actualidad existe dentro del sistema educativo un marcado desconocimiento sobre "dónde ir" en los procesos de la planificación académica que cumplen los docentes, que puede reforzarse debido a elementos de la cultura organizacional de la institución. En el esfuerzo de crear nuevas y mejores opciones para el futuro, y en la búsqueda del éxito organizacional, se suele relacionar la planificación académica con el compromiso que cada docente asume al respecto.

Sin embargo, se puede observar cómo desde la cultura instaurada, en los planteles objeto de investigación se da la existencia de una inversión de valores de quiénes dirigen y son dirigidos, es decir, el comportamiento social de la institución está mediado por las actitudes del personal y los supuestos básicos que le subyacen. Tales fallas evidencian que dentro de la cultura organizacional se han difundido valores estereotipados y supuestos contrarios a las demandas educativas en las instituciones básicas.

Al respecto, "la cultura organizacional es un factor clave para el éxito en una organización" (Gómez, 2003, p. 43). La cultura es, por lo tanto, la mayor fortaleza de una organización cuando coincide con sus estrategias, pero si esto no ocurre será una de sus principales debilidades. Conviene, entonces, afrontar la interacción que existe entre cultura organizacional y la planificación académica de una institución, para que de manera integrada las acciones, planes y proyectos alcancen la eficiencia y la competitividad organizacional necesarias.

En este orden de ideas, es de hacer notar que, luego de realizado un diagnóstico, en las Escuelas Básicas del Municipio Escolar Maracaibo $\mathrm{N}^{\circ} 5$ se observaron debilidades relacionadas con 
la planificación académica, tales como incumplimiento y extemporaneidad de las actividades académicas planificadas. Asimismo, fue posible detectar toma de decisiones ajenas a los patrones culturales de las escuelas, relacionados con los valores y supuestos básicos que caracterizan las instituciones educativas.

En este sentido, se encontró que en las instituciones objeto de estudio hay una situación similar a la planteada anteriormente, al seguir un exceso de lineamientos tradicionales directamente relacionados con la comunidad local, los padres de familia, el personal directivo y docente.

El funcionamiento de estas organizaciones está vinculado con las características que describen e identifican la situación de los sujetos adscritos a ellas en atención a su actuación, integración, motivación y comunicación, enmarcado dentro de las particularidades de una cultura organizacional inadecuada, manifestada en los procesos de planificación académica que predominan en la institución.

Pareciera que en las instituciones educativas de la región existe una planificación académica ajena a sus necesidades y características, notorias en la degradación profesional de los docentes para llevar a cabo de forma eficaz y eficiente su quehacer educativo. A las instituciones educativas se les plantea el reto de repensar su planificación académica, apoyadas en la promoción de una cultura organizacional cuyas características sean representativas de los principios filosóficos que deben dirigir las acciones y los procesos en las instituciones de educación básica; esto rescata el énfasis en la condición humana, la racionalidad de los procesos y el control social para alcanzar y dar respuesta a los cambios y transformaciones que demanda este nivel educativo, específicamente las escuelas básicas adscritas al Municipio Escolar Maracaibo $\mathrm{N}^{\circ} 5$.

Tomando en consideración las ideas expuestas, se formulan los siguientes objetivos:

\section{General:}

Analizar la relación entre la planificación académica del docente de aula y la cultura organizacional de las Escuelas Básicas del Municipio Escolar Maracaibo $\mathrm{N}^{\circ} 5$, del Estado Zulia.

\section{Específicos:}

1. Describir las fases de la planificación académica que cumplen los docentes de las Escuelas Básicas del Municipio Escolar Maracaibo $\mathrm{N}^{\circ}$ 5, del Estado Zulia.

2. Caracterizar los principios de la planificación académica que cumplen los docentes de las Escuelas Básicas del Municipio Maracaibo $\mathrm{N}^{\circ} 5$, del Estado Zulia.

3. Identificar los pasos de la planificación académica manifiestos en las Escuelas Básicas del Municipio Escolar Maracaibo $\mathrm{N}^{\circ}$ 5, del Estado Zulia.

4. Indagar los elementos básicos que intervienen en la cultura organizacional de las Escuelas Básicas del Municipio Escolar Maracaibo $\mathrm{N}^{0} 5$, del Estado Zulia.

5. Evaluar las categorías de la cultura organizacional que se manejan en las instituciones de educación básica.

6. Identificar los tipos de cultura predominantes en las Escuelas Básicas del Municipio Escolar Maracaibo $\mathrm{N}^{\circ} 5$, del Estado Zulia.

7. Establecer la relación que existe entre la planificación académica y la cultura organizacional de las Escuelas Básicas del Municipio Maracaibo $\mathrm{N}^{0} 5$, del Estado Zulia.

El cumplimiento de los objetivos reviste de importancia el estudio desde los siguientes puntos de vista: teórico, práctico, metodológico y social. En lo teórico, permitirá profundizar en la concepción sobre el deber ser de la planificación de 
la enseñanza y la cultura organizacional de las Escuelas Básicas estudiadas del Municipio Maracaibo. Desde una perspectiva metodológica, consentirá el cumplimiento de los objetivos de estudio empleando técnicas de investigación como instrumentos para medir dichas variables en las instituciones de educación básica del municipio Maracaibo.

Su valor práctico radica en que la indagación permitirá obtener resultados que pueden ser procesados de manera científica para establecer conclusiones y recomendaciones orientadas a presentar algunas alternativas de solución relacionadas con el problema. Desde el punto de vista social, contribuiría a mejorar la calidad académica a partir de los procesos de planificación de la instrucción y la cultura organizacional, hecho de gran importancia para el ámbito escolar.

\section{Planificación Académica}

La planificación académica, de acuerdo con Kaufman (2006), es una toma de decisiones donde se establecen los objetivos a lograr mediante un método o plan seleccionando las alternativas de acciones más viables para lograr las metas propuestas. Esta consiste en la elaboración de un plan donde se contemplen elementos y actividades de los agentes educativos (el alumnado, el personal docente y los grupos representantes y demás miembros de la comunidad en general), de tal manera que prevean las situaciones en las que habrá de llevarse a cabo el proceso educativo.

De igual forma, es la función a través de la cual el personal docente organiza los conocimientos, habilidades y destrezas que deberá adquirir el educando y diseña actividades educativas que estimulen el logro de aprendizajes. De esto se infiere que la planificación académica constituye la primera fase de carácter obligatorio de toda labor docente, es decir, es una exigencia a la ética profesional, un recurso para el buen control administrativo de la enseñanza.

\section{Fases de la Planificación Educativa}

Molina (2001) destaca que el docente ha de considerar como un proceso administrativo su rol de planificador de la enseñanza a partir de la vinculación entre las siguientes fases: evaluación de entrada, objetivos, selección de estrategias y retroalimentación. Ciertamente, la planificación es un proceso que vincula la interdisciplinaridad entre los elementos que la identifican.

-Evaluación de Entrada: La evaluación de entrada que se realiza al inicio del año escolar al comienzo de una unidad o bloque de objetivos permite considerar las características del educando y de cada uno de los componentes del proceso educativo, de manera que garantice el logro de los objetivos propuestos en el programa de estudio.

-Objetivos: Se refiere a las modificaciones de comportamiento que se desean obtener en el educando. En la planificación de la acción educativa se requieren objetivos precisos y claramente definidos. Para tal fin, el docente debe plantearse preguntas tales como ¿dónde se quiere llegar?, ¿qué se desea obtener?, ¿cuál es el resultado esperado?

Asimismo, se señala que "para la elaboración de los objetivos deben tomarse en cuenta enunciados que expresen el tipo de comportamiento o cambio de conducta (cognoscitivo, afectivo o psicomotor) que debe lograr el educando al finalizar el lapso de estudio" (Rosales, 2002, p. 68).

Esta posición evidencia que los objetivos específicos de aprendizaje deben reflejar precisión y exactitud describiendo el comportamiento que hará de demostrar el alumno después de haber cumplido con éxito el proceso de aprendizaje.

Tomando en consideración lo antes señalado, la especificación de los objetivos en forma clara, precisa y concreta, evita que la enseñanza se lleve a cabo en forma intuitiva, desorganizada e improvisada. 
-Realimentación: En esta fase se prevén criterios de evaluación a aplicar durante el desarrollo del plan y al final del mismo, con la finalidad de obtener resultados que permitan realizar una labor de constante reajuste para reformular, si fuera necesario, las distintas fases del plan (realimentación) y mejorar la elaboración de futuros planes. Esta posición es compartida por Rosales (2002), quien afirma que la realimentación es la última fase de la planificación académica y es la que indica si se debe continuar, modificar o si se lograron los objetivos. Para ello el docente debe diseñar criterios de evaluación con instrumentos pertinentes que recojan la información para la toma de decisiones en forma acertada.

En virtud de lo expuesto, cabe plantear que esta fase permite la flexibilidad de la evaluación del proceso de planificación académica. Asimismo, se destaca que los procesos de la planificación académica están inmersos en un enfoque sistémico que atienden cada uno de los momentos que la caracterizan.

\section{Principios de la Planificación Académica}

La planificación, según Melinkoff (2004), tiene como base una serie de principios que se analizan a continuación:

-Flexibilidad: todo plan o programa debe ser flexible; ante cualquier eventualidad que se presente en la ejecución del mismo, debe preverse mecanismos de ajustes y de adaptación o cambios en las condiciones sociales, económicas o de otra naturaleza. La flexibilidad debe dotar a la planificación de alternativas que garanticen la culminación y logros de los objetivos propuestos y, por ende, del propósito de la planificación académica.

-Unidad: los planes deben tener una integración orgánica, armónica y coherente a objeto de obviar la duplicidad de esfuerzos y el mal uso de los recursos. Quizás uno de los problemas más evidentes en la institución es la planificación incoherente, carente de ilación, armonía entre la variedad de proyectos, planes y programas, que al fin y al cabo persiguen un mismo objetivo; esta incoordinación evidente conlleva al docente a tener una actitud de rechazo o cualquier cambio que se quiera realizar en el campo institucional.

-Previsión: se basa en que los planes deben presentarse en los lapsos definidos en que se ejecutarán las diferentes actividades; así mismo, se deberán prever en la institución y jerarquizar los recursos necesarios para su realización.

Los principios de la planificación académica en la educación básica se apoyan en la acción curricular para prever acciones dirigidas al fortalecer la práctica pedagógica, la investigación en la acción docente y el diagnóstico de la acción académica para extraer los cursos de alternativa de acción. Para ello, se identifican los principios y se comparan con los objetivos y metas para elaborar los planes curriculares, en atención a las oportunidades y fortalezas del alumno, del docente, del plantel, del entorno; esto permite generar los presupuestos que determinarán los insumos necesarios para la aplicación de los planes curriculares.

Asimismo, se parte de la premisa que los principios de planificación académica y la acción curricular necesitan de la implementación de mecanismos de coordinación y de un nivel de satisfacción laboral del docente que eleve su disposición hacia el proceso en cada uno de los pasos de la planificación académica.

\section{Pasos de la Planificación como Proceso}

Al planificar se está dando lugar a una serie de situaciones que giran en torno al ser humano, dichas situaciones pueden ser adversas y ameritan de ciertas decisiones que las modifiquen a fin de adecuarlos a las potencialidades de las instituciones. 
Sin embargo, cualquier decisión sobre planificación y los criterios pertinentes no pueden ser tomados a la ligera; ameritan según Stoner, Freeman y Gilbert (2004) una serie de pasos a considerar en la planificación. Deallí, que serán considerados los siguientes: formulación de metas, identificación de los actuales objetivos, análisis de recursos, identificación de oportunidades, estrategias y determinación del grado de cambio adquirido o requerido.

A continuación, se hará un recuento o análisis referente a cada uno de estos aspectos:

-Formulación de Metas: "la planificación de metas implica comprender la misión de la organización y después establecer metas que la traduzcan a términos concretos" (Stoner et ál., 2004, p. 200). En consecuencia, las metas señalan la índole e intencionalidad del esfuerzo del plan que se ha emprendido en un período de tiempo determinado, en función de las necesidades y objetivos planteados.

Por otro lado, el mismo autor señala que las metas proporcionan un sentido de dirección, y sin una los individuos al igual que las organizaciones tienden a la confusión y reaccionan ante los cambios del entorno sin un sentido claro de lo que en realidad quieren alcanzar. Al establecer las metas, la gerencia y las organizaciones refuerzan su motivación y encuentran una fuerte inspiración que los ayuda a rebasar los inevitables obstáculos que encuentran.

-Identificación de los actuales Objetivos y Estrategias: La identificación de objetivos y estrategias es indispensable para precisar la forma en que se satisfacen las necesidades diagnosticadas y cómo se cumplirán los mandatos legales que definen los fines propuestos por una gestión o un director en las escuelas básicas.

Según Castellano (2001), la identificación de objetivos se asocia con aquello que se desea alcanzar mediante acciones propuestas por el proceso de planificación. Las fuentes de los objetivos y estrategias son múltiples, pero es posible distinguir tres tipos primordiales: primero, aquellos previamente definidos en las leyes, reglamentos o estatutos; segundo, aquellos que se hacen evidentes en virtud de una problemática observable; y tercero, aquellos que resultan de diagnósticos dentro de un proceso de planificación preventiva a mayor o menor plazo.

-Análisis de Recursos: Los recursos se asocian con medios materiales, humanos y técnicos que representan un marco de referencia para analizar los logros del plantel. "Los recursos son todos los instrumentos que utiliza el orientador o docente para el desarrollo de los objetivos en el proceso enseñanzaaprendizaje" (Venezuela. Ministerio de Educación, 2005, p. 64).

Asimismo, se señala que "los recursos son necesarios para determinar sus ventajas y desventajas competitivas. Unas y otras se refieren a las fortalezas y debilidades de la organización frente a sus competidores actuales y futuros" (Stoner et ál, 2004, p. 203).

En el caso específico de la gerencia educativa en las escuelas básicas, esta necesita estar al tanto de los recursos disponibles y de lo que hace falta; también analizar sus ventajas y desventajas y la relación que guardan con los objetivos y estrategias.

Es de destacar que los recursos se asocian con las personas, herramientas, objetos o eventos que proporcionan el apoyo necesario para facilitar o desarrollar una tarea. En este orden, el término recurso se refiere a todos los medios físicos o humanos que representan el conjunto total de condiciones de trabajo que hacen posible los objetivos en un mínimo de tiempo y esfuerzo.

-Identificación de los Riesgos: Smith (2002) plantea que: "El riesgo se asocia con la personalidad y condiciones de trabajo. La condición es mantener el mínimo de riesgo lo que puede lograrse con la planificación juiciosa y con el uso de control formativo y preventivo. El gerente no debe conformarse con solo dejar que las cosas sucedan. Tiene que dar los pasos 
necesarios para conformar el futuro que él quiera" (p. 43).

Existe una gran diferencia entre reconocer la posible ocurrencia de riesgo y dar los pasos para asegurarse de que tales riesgos no cobrarán realidad. Es evidente que ejecutar una acción no evita que se produzca el riesgo, sino que permite prepararse para ello y tener pleno control sobre la situación.

Stoner et ál. (2004) refiere que, para la identificación de estrategias, el análisis del ambiente y el de los recursos de la organización se combinan para descubrir las oportunidades disponibles para la organización y los riesgos que se puedan enfrentar.

Por consiguiente, al planificar se deben considerar aquellas situaciones de riesgos dentro de la organización para que estratégicamente se puedan controlar en beneficio institucional, siéndose muy cuidadoso ante las situaciones de riesgo que pudieran generar algún daño físico, psíquico u organizacional.

-Determinación del Grado de Cambio Requerido: Es de sumo interés para cualquier docente al planificar ya que determina el grado de cambio que se espera lograr; dependerá del ambiente o clima organizacional y de la efectividad de la estrategia seleccionada. Ahora bien, son muchas las fuerzas que llevan a las organizaciones a cambiar dado que existen fuerzas encontradas que actúan para mantener a la organización en un estado de equilibrio. En consecuencia, la determinación del grado de cambios requeridos es un fenómeno que presenta un reto sin precedentes para el docente y por ende es propio de las organizaciones educativas, las cuales deben modificar las estrategias en el tiempo. Debido a esto, los gerentes tienen que manejar la resistencia.

\section{Cultura Organizacional}

La cultura puede inferirse de lo que los empleados dicen, hacen y piensan dentro de una situación organizacional, implica el aprendizaje y transmisión de conocimientos, creencias y patrones de conducta durante un período. En relación con esta posición, se plantea que "la cultura es el patrón que genera conductas, creencias compartidas y valores que los miembros tienen en común" (Koontz y Heinz, 2002, p. 61). Por su parte, Shein (2004) afirma que la cultura es la filosofía de una organización o institución que ha adoptado en base a supuestos, conductas, relatos, mitos y otros que la definen como tal. El mismo autor señala que existen dentro de este modelo tres elementos o niveles básicos de la cultura: los artefactos, los valores adoptados y los supuestos básicos.

-Artefactos: Son aspectos que se ven, escuchan y sienten cuando un individuo se encuentra ante un grupo nuevo que tiene una cultura desconocida para él. En este sentido, Shein (2004) expresa que estos incluyen conductas, servicios y productos de los miembros del grupo que se toman en consideración al ver las manifestaciones, aportes y producciones, con lo que se puede observar la cultura organizativa (distintivos, símbolos, logotipos, lenguaje, banderas, espacios, colores, etc.) (p. 37). Cabe destacar que en este nivel se realizan creaciones o producciones de los actores como forma de proyección para que se sientan vinculados a su lugar de trabajo específico.

-Valores Adoptados: En relación a los valores adoptados, Shein (2004) señala que estos son utilizados para explicar la forma en que y por qué se hace lo que se hace mediante la integración y el consenso en la búsqueda del logro de los objetivos, lo que es comprobable al estudiar comportamiento de los actores y el resultado de las actividades.

Así mismo, se plantea que este nivel justifica los nuevos enfoques de cultura organizacional elegidos, facilitando así la integración de personas en los procesos para la consecución de los objetivos mediante un seguimiento o control de todas las actividades programadas a fin de realizar las correcciones pertinentes. 
Igualmente, Robbins (2001) afirma que estos son conceptos básicos y creencias que definen el éxito en términos concretos para los empleados. Así mismo, representan la base de evaluación que los miembros de una organización emplean para juzgar situaciones, actos, objetos y personas. Estos reflejan las metas reales y la misión de la institución.

Además, los valores adoptados establecen normas para la organización. Son la esencia de la filosofía que la institución tiene para alcanzar el éxito. De esta forma, proporcionan un sentido de dirección común para todos los empleados y establecen las directrices para su compromiso diario. Por lo tanto, toda organización con aspiraciones de excelencia debería tener comprendidos y sistematizados los valores y las ideas que constituyen el comportamiento motor de la organización, en especial la de educación superior, si se parte del principio que dicha institución es la mejor en la prestación de servicio.

-Supuestos Básicos: Se define este nivel como un patrón que un determinado grupo ha creado o inventado con el propósito de hacerle frente a los problemas de adaptación externa e integración, el cual ha funcionado muy bien para considerarse válido cada vez que se presenten problemas similares.

Bajo esta premisa, la cultura es "la manera indicada de hacer las cosas muchas veces por supuestos implícitos" (Bueno, 2003, p. 110). Estos supuestos corresponden a la filosofía o bases explicativas que sustentan el porqué de la cultura y de su función en la organización. También debe reflejarse en este nivel la naturaleza humana y su relación con el contorno o realidad vital, que vienen a caracterizar las culturas de las organizaciones.

\section{Características de las Culturas Organizacionales}

Las organizaciones, al igual que las huellas digitales, son siempre singulares.
Cada una posee su propia historia, patrones de comunicación, sistemas y procedimientos, declaraciones de filosofía, historia y mitos que, en su totalidad, constituyen su cultura. Algunas presentan un ambiente de mucho dinamismo, otras tienen un entorno de tranquilidad.

Bueno (2003) establece que las culturas de unas organizaciones pueden ser amables y amistosas, mientras que otras dan la impresión de ser frías y asépticas. Con el tiempo, la cultura de la organización llega a ser conocida por los empleados y el público. Cabe destacar que la cultura se perpetúa, porque la organización tiende a atraer y conservar a individuos que parecen aceptar sus valores y creencias.

-Cultura de Poder: supone que las organizaciones requieren de líderes fuertes que puedan manejar las desigualdades que se presentan y mantener el equilibrio general del sistema. Al respecto, Koontz y Heinz (2002) sostiene que "los recursos que serán distribuidos de manera desigual son: el dinero, el privilegio, la seguridad y calidad general de vida" (p. 78). En las organizaciones con fuerte cultura de poder, existe un clima propicio para el desarrollo de actividades que allí se llevan a cabo, sus líderes son firmes pero equitativos y generosos con sus seguidores; por lo tanto, existe una aceptación de la estructura de la organización.

-Cultura de Rol: Koontz y Heinz (2002) afirma que está constituida de manera racional por estructuras y sistemas derivados del poder puro. Su basamento es que el trabajo se logra a través de la regulación de la ley. El mismo autor la define como el desarrollo de los roles, las responsabilidades y las retribuciones potenciales de cada miembro de la organización que especifican sus asignaciones, al mismo tiempo que se instalan los sistemas apropiados para asegurar que el cumplimiento de roles se lleve a cabo de manera justa.

Esta cultura tiene una ventaja muy importante, y es que maneja adecuadamente la estabilidad, justicia y eficiencia. Los 
miembros de la organización se sienten protegidos de decisiones arbitraras que se tomen en los altos niveles y, en consecuencia, pueden utilizar sus energías en las tareas en lugar de preocuparse por la autoprotección.

-Cultura de Logro: su supuesto básico hace referencia a las contribuciones significativas que hacen las personas a su trabajo y a la sociedad en general, y de esta manera disfrutan su interrelación con los clientes y demás compañeros de trabajo. En este sentido, Stoner et ál. (2004) la concibe como la satisfacción de una labor cumplida y bien realizada y de interacción significativa entre los compañeros de un lugar de trabajo y que constituye las verdaderas retribuciones o recompensas intrínsecas y de las interacciones satisfactorias.

-Cultura de Apoyo: se fundamenta en el supuesto básico de la enseñanza y el apoyo mutuo, los cuales deben constituir la base fundamental de las relaciones entre las organizaciones y sus miembros. En tal sentido, Bueno (2003) señala que las personas deben ser consideradas y valoradas como seres humanos y no solo porque contribuyen con el trabajo que se ha asignado o porque ocupan roles organizacionales.

De esta manera, se establecen metas más estrechas, con calidad para los clientes y demás personas del público. Las organizaciones que están bajo este tipo de cultura se caracterizan por practicar una comunicación abierta, sincera y de apoyo, y sus relaciones interpersonales son bastante estrechas. La armonía constituye un valor importante y se evitan las confrontaciones que puedan destruirla.

\section{Tipos de Cultura}

El análisis previsto indica discrepancia con Gibson y Donnely (2001), quienes consideran que "la cultura organizacional provee de patrones y expectativas previsibles a las organizaciones, permitiéndoles resolver problemas, cumplir metas y relacionarse con clientes importantes, proveedores y otros grupos interesados"(p. 123).

Dentro de una organización puede existir más de una cultura organizacional, y a cada variación se le denomina subcultura, las cuales surgen dentro de pequeños grupos de trabajo, entre las que se destacan según el mismo autor.

-Cultura Burocrática: son aquellas cuyos empleados aprecian la formalización, las reglas, los procedimientos estándar de operación y la coordinación jerárquica, y valoran altamente la estandarización de los bienes y del servicio al cliente. Los gerentes conciben su papel como el de coordinadores, organizadores y vigilantes del cumplimiento de las reglas y normas.

Las tareas y autoridad de todos los empleados están claramente definidas y son típicamente conservadoras, tradicionales y dirigidas burocráticamente. Se espera que las personas se adapten y sigan las normas.

-Cultura del Clan: Para Shein (2004), la cultura del clan es propia de instituciones que buscan el control interno pero con flexibilidad y donde están preocupados por sus empleados y muestran sensibilidad a los clientes. Esta se caracteriza por estar orientada "hacia dentro" o ser un sistema cerrado en lugar de "hacia el exterior". La dirección de este tipo de empresas es muy controladora, supervisando muy de cerca a los trabajadores, quienes son capaces de ejecutar las órdenes sin preguntar.

De igual manera, se consideran introvertidas, inflexibles, cargadas de viejas tradiciones y resistentes al cambio. En este tipo de cultura se regula el comportamiento de los empleados mediante valores compartidos, normas tradiciones, rituales, creencias y otros aspectos de la cultura de la organización.

-Cultura del Emprendedor: es la organización preparada para iniciar, comenzar o hacer actividades previamente establecidas donde, según Prieto (2002), hay que tener una actitud emprendedora y el espíritu emprendedor es inherente al ser 
humano y evoluciona. El emprendedor no debe tener dificultad para comunicarse con los demás.

Este tipo de cultura necesita desarrollar una mentalidad emprendedora y estratégica para sobrevivir y crear valor. Por esta razón y particularmente en entornos dinámicos e inciertos, es importante la convergencia entre los avances de la dirección estratégica con la capacidad de detección de las oportunidades, así como para actuar y movilizar recursos. En este sentido, agrega el autor citado, la teoría basada en los recursos puede ser útil para avanzar en esta confluencia, al tiempo que permite desarrollarlos.

Crear una cultura emprendedora implica, en primer lugar, un convencimiento por parte de la alta dirección de que eso es importante, la cual ha de entender que esto implica soltar la iniciativa en busca de oportunidades y que eso solo es posible con un sistema de control flexible.

\section{Metodología}

La investigación se realizó bajo un enfoque positivista, que afirma que en la realidad existe un orden único que tiende al progreso indefinido de la sociedad (Hernández, Fernández y Batista, 2006).

La tipificación de una investigación se establece de acuerdo con el tipo de problema que se desea solucionar, los objetivos que se pretenden lograr y la disponibilidad de recursos necesarios para la consecución de la meta propuesta (Chávez, 2004). En tal sentido, el tipo de investigación que se utilizó fue el explicativo en su modalidad de campo. Los datos se recolectaron de manera directa dentro de la realidad en la que se inserta la problemática, sin manipular o controlar variable alguna.

La población de estudio está conformada por 156 personas, distribuidos en 143 docentes y 13 directivos, en 6 escuelas de educación básica del Municipio Escolar Maracaibo $\mathrm{N}^{\circ} 5$ y la muestra estuvo representada por un total de 106 sujetos. La técnica de recolección de datos fue la encuesta y como instrumento se utilizó un cuestionario con una escala tipo Likert.

Encuanto a la validez delinstrumento, se sometió a la validez de contenido y de constructo. Para la confiabilidad del instrumento se utilizo el coeficiente de Alfa Cronbach, se obtuvo un valor de 0,99 evaluándolo como altamente confiable.

\section{Resultados y Discusión}

Los resultados aportados por la muestra total considerada en el estudio se analizaron mediante el paquete estadístico Statistical Packege for Social Sciences (SPSS); se aplicó la estadística descriptiva media, desviación estándar para cada una de las variables, dimensiones e indicadores, utilizando los baremos establecidos para analizar la cultura organizacional y planificación académica del docente educativo, así como las frecuencias absolutas y relativas de las respuestas aportadas en cada una de las alternativas.

-El cálculo estadístico del chi cuadrado señaló que existe una relación altamente significativa entre planificación académica y cultura organizacional, al observarse que la probabilidad calculada es menor que la establecida (Ver tabla 1). Este resultado evidencia que la cultura organizacional predominante en la institución está afectando la planificación académica de los docentes.

Tabla 1

Distribución de la relación chi cuadrado entre las variables planificación académica y cultura organizacional

\begin{tabular}{lc}
\hline Posición & Valor \\
\hline Probabilidad Establecida & 0.05 \\
Probabilidad Calculada & 0.0042 \\
Grados de Libertad & 3 \\
Casos & 106 \\
\hline
\end{tabular}

Fuente: Ojeda y Ferrer (2010). 
La variable planificación académica, de acuerdo con los resultados de su análisis frecuencial, indica que el $30 \%$ de los encuestados señalaron estar ni de acuerdo ni en desacuerdo en que se cumple con la planificación académica en las instituciones de educación básica investigadas; un 25\% y $23 \%$ de los docentes entrevistados indicaron estar de acuerdo y en desacuerdo respectivamente. Asimismo, se indicó una media de 3,641, señalando que existe acuerdo para lograr cumplir con la planificación académica dentro delasinstituciones investigadas. La desviación estándar con un valor $\pm 1,321$ indica que la media puede variar entre las alternativas de acuerdo y en desacuerdo. La mediana con un valor de 3,900 se ubicó por encima de la media evidenciando una tendencia de opiniones hacia las alternativas altas de medición (Ver tabla 2).

Tabla 2

Análisis frecuencial descriptivo de la variable planificación académica

\begin{tabular}{lcccc}
\hline Alternativas & Frecuencia & $\%$ & Fracuencia acumulada & \% acumulado \\
\hline Totalmente de acuerdo & 14 & 13 & 14 & 13 \\
De Acuerdo & 26 & 25 & 40 & 38 \\
Ni de acuerdo ni en desacuerdo & 31 & 30 & 71 & 98 \\
En desacuerdo & 25 & 23 & 96 & 100 \\
Totalmente en desacuerdo & 10 & 9 & 3,641 & 1,321 \\
\hline Media & & 3,9000 & \\
\hline Desviación Estándar & & \\
\hline Mediana & & & \\
\hline
\end{tabular}

Fuente: Ojeda y Ferrer (2010).

Al respecto, es de destacar que la planificación académica, de acuerdo con Kaufman(2006), es una toma de decisiones donde se establecen los objetivos a lograr mediante un método o plan seleccionando las alternativas de acciones más viables para lograr las metas propuestas.

En relación con la dimensión fases de la planificación académica, como producto del análisis conjunto de los indicadores evaluación de entrada, objetivos, selección de estrategias y retroalimentación, se indica que el $34 \%$ de las personas encuestadas señalaron estar ni de acuerdo ni en desacuerdo en que se cumple con las fases de la planificación académica; un $28 \%$ y $19 \%$ de los docentes entrevistados indicaron estar de acuerdo y en desacuerdo respectivamente. Cabe destacar que los menores porcentajes se indicaron para las alternativas extremas de totalmente de acuerdo y totalmente en desacuerdo. Asimismo, se indicó una media de 3,0115, que corrobora la existencia de un ni acuerdo ni desacuerdo para lograr cumplir con las fases de la planificación académica. La desviación estándar con un valor $\pm 1,0342$ indica que la media puede variar entre las alternativas de acuerdo y totalmente de acuerdo. Por otro lado, la mediana con un valor de 4,4000 se ubicó por encima de la media evidenciando una tendencia de opiniones hacia las alternativas altas de medición (Ver tabla 3).

Sin embargo, Molina (2001) destaca que el docente ha de considerar como un proceso administrativo su rol de planificador de la enseñanza, a partir de la vinculación entre las distintas fases de la planificación académica. 
Tabla 3

Análisis frecuencial descriptivo de la dimensión fases de la planificación académica

\begin{tabular}{lcccc}
\hline Alternativas & Frecuencia & $\%$ & Frecuencia acumulada & \% acumulado \\
\hline Totalmente de acuerdo & 15 & 14 & 15 & 14 \\
De acuerdo & 30 & 28 & 45 & 42 \\
Ni de acuerdo ni en desacuerdo & 36 & 34 & 81 & 76 \\
En desacuerdo & 20 & 19 & 101 & 95 \\
Totalmente en desacuerdo & 5 & 5 & 106 & 100 \\
\hline Media & & & 3,0115 & \\
\hline Desviación Estándar & & & \\
\hline Mediana & & & \\
\hline
\end{tabular}

Fuente: Ojeda y Ferrer (2010).

En cuanto a la dimensión principios de la planificación académica, como producto del análisis conjunto de los indicadores flexibilidad, unidad y previsión, se indica que el $27 \%$ de los encuestados señalaron estar en desacuerdo en que se cumple con los principios de la planificación académica; un $26 \%$ y $21 \%$ de los docentes entrevistadas indicaron estarnideacuerdo ni en desacuerdo y totalmente de acuerdo respectivamente. Asimismo, se obtuvo una media de 4,1450, implicando que existe acuerdo para lograr cumplir con la dimensión principios de la planificación académica. La desviación estándar con un valor $\pm 1,0540$ indica que la media puede variar entre las alternativas de acuerdo y desacuerdo. Por otro lado, la mediana con un valor de 4,5000 se ubicó por encima de la media evidenciando una tendencia de opiniones hacia las alternativas altas de medición (Ver tabla 4).

En este sentido, Melinkoff (2004) indica que los principios de la planificación académica en la educación básica se apoyan en la acción curricular para prever acciones dirigidas al fortalecer: la práctica pedagógica, la investigación en la acción docente y el diagnóstico de la acción académica para extraer los cursos de alternativa de acción.

Tabla 4

Análisis frecuencial descriptivo de la dimensión principios de la planificación académica

\begin{tabular}{lcccc}
\hline Alternativas & Frecuencia & $\%$ & Frecuencia acumulada & \% acumulado \\
\hline Totalmente de acuerdo & 22 & 21 & 22 & 21 \\
De acuerdo & 20 & 19 & 42 & 40 \\
Ni de acuerdo ni en desacuerdo & 28 & 26 & 69 & 66 \\
En desacuerdo & 29 & 27 & 99 & 93 \\
Totalmente en desacuerdo & 7 & 7 & 106 & 100 \\
\hline Media & & 4,1450 & 1,0540 \\
\hline Desviación Estándar & & 4,5000 & \\
Mediana & &
\end{tabular}

Fuente: Ojeda y Ferrer (2010). 
La dimensión pasos de la planificación académica se analizó en forma conjunta con los indicadores formulación de metas, identificación de los objetivos y estrategias, análisis de recursos, identificación de riesgos y determinación del grado de cambio requerido, y en ella se indica que el $27 \%$ de los encuestados señalaron estar ni de acuerdo ni en desacuerdo en que se cumplen con los pasos de la planificación académica; un $26 \%$ y $23 \%$ de los docentes entrevistados indicaron estar totalmente de acuerdo y en desacuerdo respectivamente. Asimismo, se indicó una media de 4,2450 , señalando que existe acuerdo para lograr cumplir con la dimensión pasos de la planificación académica. La desviación estándar con un valor $\pm 1,3531$ indica que la media puede variar entre las alternativas de acuerdo y en desacuerdo. Por otro lado, la mediana con un valor de 4,1000 se ubicó por debajo de la media evidenciando una tendencia de opiniones hacia las alternativas bajas de medición (Ver tabla 5).

Sin embargo, según Stoner et ál (2004) cualquier decisión sobre planificación y los criterios pertinentes, a cada uno de los pasos que ella cumple, no puede ser tomada a la ligera.

Tabla 5

Análisis frecuencial descriptivo de la dimensión pasos de la planificación académica

\begin{tabular}{lcccc}
\hline Alternativas & Frecuencia & $\%$ & Fracuencia acumulada & $\%$ acumulado \\
\hline Totalmente de acuerdo & 5 & 5 & 5 & 5 \\
De acuerdo & 28 & 26 & 33 & 31 \\
Ni de acuerdo ni en desacuerdo & 29 & 27 & 62 & 58 \\
En desacuerdo & 24 & 23 & 86 & 100 \\
Totalmente en desacuerdo & 20 & 19 & 106 & 4,2450 \\
\hline Media & & 1,3531 & 4,1000 \\
\hline Desviación Estándar & &
\end{tabular}

Fuente: Ojeda y Ferrer (2010).

La variable cultura organizacional, se analizóa partir delas dimensiones elementos, categorías y tipos, en la que se indica que el $29 \%$ de los encuestados, señalaron en estar ni de acuerdo ni en desacuerdo en que existe una cultura organizacional instaurada en las instituciones investigadas; un $25 \%$ y $24 \%$ de los docentes entrevistadas indicaron estar en desacuerdo y de acuerdo respectivamente. Asimismo, se indicó una media de 3,474, señalando que existe acuerdo para reconocer la cultura organizacional instaurada en las instituciones investigadas. La desviación estándar con un valor $\pm 1,0064$ indica que la media puede variar entre las alternativas de acuerdo y en desacuerdo. Por otro lado, la mediana con un valor de 3.312 se ubicó por debajo de la media evidenciando una tendencia de opiniones hacia las alternativas bajas de medición. (Ver tabla 6).

Los resultados expuestos, contradicen la posición de Koontz y Heins (2002), quien expresa que la cultura puede inferirse de lo que los empleados dicen, hacen y piensan dentro de una situación organizacional e implica el aprendizaje y transmisión de conocimientos, creencias y patrones de conducta durante un período. 
Tabla 6

Análisis frecuencial descriptivo de la variable cultura organizacional

\begin{tabular}{lcccc}
\hline Alternativas & Frecuencia & $\%$ & Frecuencia acumulada & \% acumulado \\
\hline Totalmente de acuerdo & 14 & 13 & 14 & 13 \\
De acuerdo & 25 & 24 & 39 & 37 \\
Ni de acuerdo ni en desacuerdo & 31 & 29 & 70 & 96 \\
En desacuerdo & 26 & 25 & 96 & 100 \\
Totalmente en desacuerdo & 10 & 9 & 3,474 & 106 \\
\hline Media & & 3,0064 & \\
\hline Desviación Estándar & &
\end{tabular}

Fuente: Ojeda y Ferrer (2010).

En relación con la dimensión elementos de la cultura organizacional, en la que se analizaron losindicadores artefactos, valores adoptados y supuestos básicos, se indicó que el $28 \%$ de los encuestados señalaron estar ni de acuerdo ni en desacuerdo en que se cumple con los elementos de la cultura organizacional; un $25 \%$ y $22 \%$ de los docentes entrevistados indicaron estar en desacuerdo y de acuerdo respectivamente. Asimismo, se indicó una media de 3,844, señalando que existe acuerdo para lograr cumplir con la dimensión elementos de la cultura organizacional. La desviación estándar con un valor $\pm 1,141$ indica que la media puede variar entre las alternativas de acuerdo y en desacuerdo. La mediana con un valor de 2,988 se ubicó por debajo de la media evidenciando una tendencia de opiniones hacia las alternativas bajas de medición (Ver tabla 7).

$\mathrm{Al}$ respecto, Shein (2004) afirma que la cultura es la filosofía de una organización o institución que ha adoptado en base a supuestos, conductas, relatos, mitos y otras que la definen como tal.

Tabla 7

Análisis frecuencial descriptivo de la dimensión elementos de la cultura organizacional

\begin{tabular}{lcccc}
\hline Alternativas & Frecuencia & $\%$ & Frecuencia acumulada & \% acumulado \\
\hline Totalmente de acuerdo & 20 & 19 & 20 & 19 \\
De acuerdo & 23 & 22 & 43 & 41 \\
Ni de acuerdo ni en desacuerdo & 30 & 28 & 73 & 69 \\
En desacuerdo & 26 & 25 & 99 & 94 \\
Totalmente en desacuerdo & 7 & 6 & 106 & 100 \\
\hline Media & & 3,844 & 1,141 \\
\hline Desviación Estándar & & 2,988 & \\
\hline Mediana & &
\end{tabular}

Fuente: Ojeda y Ferrer (2010). 
Para la dimensión categorías como producto del análisis conjunto de los indicadores cultura del poder, cultura de rol, cultura de logro y cultura de apoyo, se señala que el $28 \%$ de los encuestados consideran estar ni de acuerdo ni en desacuerdo en que se cumple con las categorías de la cultura organizacional; un $25 \%$ y $22 \%$ de los docentes entrevistados indicaron estar de acuerdo y en desacuerdo respectivamente. Asimismo, se indicó una media de 3,2944, señalando que existe acuerdo para lograr cumplir con la dimensión categorías de la cultura organizacional. La desviación estándar con un valor $\pm 1,0014$ indica que la media puede variar entre las alternativas de acuerdo y en desacuerdo. Por otro lado, la mediana con un valor de 3,0020 se ubicó por debajo de la media evidenciando una tendencia de opiniones hacia las alternativas bajas de medición (Ver tabla 8).

Sin embargo, Bueno (2003) establece que las culturas de las organizaciones pueden ser amables y amistosas; mientras que otras dan la impresión de ser frías y asépticas. Con el tiempo, la cultura de la organización llega a ser conocida por los empleados y el público.

Tabla 8

Análisis frecuencial descriptivo de la dimensión categorías de la cultura organizacional

\begin{tabular}{lcccc}
\hline Alternativas & Frecuencia & $\%$ & Frecuencia acumulada & $\%$ acumulado \\
\hline Totalmente de acuerdo & 7 & 6 & 7 & 6 \\
De acuerdo & 23 & 22 & 30 & 28 \\
Ni de acuerdo ni en desacuerdo & 30 & 28 & 60 & 86 \\
En desacuerdo & 26 & 25 & 86 & 100 \\
Totalmente en desacuerdo & 20 & 19 & 3,2944 & 106 \\
\hline Media & & 3,0014 & \\
\hline Desviación Estándar & & 3,0020 & \\
\hline Mediana & &
\end{tabular}

Fuente: Ojeda y Ferrer (2010).

La dimensión tipos de cultura organizacional se analizó en forma conjunta con sus indicadores burocrática, clan y emprendedora, y se encontró que el $28 \%$ de los encuestados señalaron en estar de acuerdo en que se cumple con los tipos de cultura organizacional; un $27 \%$ y $26 \%$ de los docentes entrevistados indicaron estar ni de acuerdo ni en desacuerdo respectivamente. Asimismo, se indicó una media de 3,2114, implicando que existe acuerdo para lograr cumplir con la dimensión tipos de cultura organizacional. La desviación estándar con un valor $\pm 1,1144$ indica que la media puede variar entre las alternativas de acuerdo y en desacuerdo. La mediana con un valor de 3,8200 se ubicó por encima de la media evidenciando una tendencia de opiniones hacia las alternativas altas de medición (Ver tabla 9).

El análisis previsto indica discrepancia con Gibson, Ivancevich, Donnely (2001), quienes consideran que la cultura organizacional provee de patrones y expectativas previsibles a las organizaciones, permitiéndoles resolver problemas, cumplir metas y relacionarse con clientes importantes, proveedores y otros grupos interesados. 
Tabla 9

Análisis frecuencial descriptivo de la dimensión tipos de cultura organizacional

\begin{tabular}{lcccc}
\hline Alternativas & Frecuencia & $\%$ & Frecuencia acumulada & \% acumulado \\
\hline Totalmente de acuerdo & 15 & 14 & 15 & 14 \\
De acuerdo & 30 & 28 & 45 & 42 \\
Ni de acuerdo ni en desacuerdo & 29 & 27 & 74 & 69 \\
En desacuerdo & 27 & 26 & 101 & 95 \\
Totalmente en desacuerdo & 5 & 5 & 106 & 100 \\
\hline Media & & 3,2114 & \\
\hline Desviación Estándar & & & 1,1144 \\
\hline Mediana & & 3,8200 & \\
\hline
\end{tabular}

Fuente: Ojeda y Ferrer (2010).

\section{Conclusiones}

- En relación con el objetivo de determinar la relación entre la planificación académica y la cultura organizacional se determinó que existe una correspondencia de dependencia entre las variables evaluadas, evidenciándose que la planificación académica está siendo afectada o varía según la cultura organizacional imperante en las instituciones investigadas.

- En cuanto al objetivo que refiere describir las fases de la planificación académica, se constató que existe un ni de acuerdo ni en desacuerdo en que se cumple; solo el $28 \%$ de los docentes entrevistadas indicaron estar de acuerdo. Asimismo, el valor de la media confirmó estadísticamente la opinión de los investigados.

- Se evidenció entre los investigados, en atención al objetivo que reseña caracterizar los principios de la planificación académica, desacuerdos en el cumplimiento de los mismos, específicamente en atención a los principios de flexibilidad, unidad y previsión, dado que no se plantean la continuidad, modificación y logro de los objetivos institucionales.
- En relación con el objetivo de identificar los pasos de la planificación académica, se pudo afirmar que existe un ni de acuerdo ni en desacuerdo con el cumplimiento de los mismos, manifiesto en la poca consideración en la identificación de los actuales objetivos y estrategias, el análisis de recursos y la determinación del grado de cambio requerido. En este sentido, no existe un total acuerdo de cumplimiento de los pasos de una planificación académica, implicando que el personal adscrito a las instituciones evaluadas está al margen de las ventajas y desventajas competitivas de una planificación académica, desconociendo sus fortalezas y debilidades de la organización frente a las necesidades sociales actuales y futuros.

- En líneas generales, la planificación académica ejercida por los docentes investigados no se realiza con la debida efectividad y eficiencia, en tanto que están ni de acuerdo ni en desacuerdo en que se dé una evaluación de entrada y selección de estrategias.

- De igual manera, se pudo establecer en relación al objetivo que revela indagar los elementos básicos que intervienen en 
la cultura organizacional que existe un ni de acuerdo ni en desacuerdo entre el personal, en concordancia con la identificación de las características propias de la organización, las cuales identifican a las instituciones educativas de acuerdo a normas, valores, mitos, estereotipos, jerarquías, conflictos, intereses y creencias.

- En atención al objetivo que permitió evaluar las categorías de la cultura organizacional que predominan en las instituciones de educación básica, se pudo evidenciar que el $28 \%$ de los encuestados señalaron estar ni de acuerdo ni en desacuerdo en que se cumpla. Específicamente, al propiciar el manejo de las desigualdades que se presentan entre el personal, pues consideran que se presentan abusos institucionales por parte de los líderes para sacar ventaja. Es necesario que se proporcionen sistemas de control apropiados que aseguren el cumplimiento de los roles culturales de manera justa y así los docentes se sientan protegidos de las decisiones arbitrarias que se toman dentro de la institución.

- En cuanto al objetivo que permitió identificar los tipos de cultura organizacional, se constató que el $28 \%$ de los encuestados señalaron en estar de acuerdo en que se da una cultura organizacional burocrática, de clan y emprendedora; un 27\% y $26 \%$ de los docentes entrevistados indicaron estar ni de acuerdo ni en desacuerdo respectivamente. Asimismo, se obtuvo una media de 3.2114, implicando que existe acuerdo para lograr cumplir con la dimensión tipos de cultura organizacional.

- Fue posible detectar coincidencia de opiniones en estar de acuerdo con que en las instituciones investigadas la cultura organizacional provee de patrones y expectativas previsibles dentro de la organización, los que determinan la forma de resolver problemas, el cumplimiento de metas y el trato entre el personal.

\section{Referencias bibliográficas}

Ander-Egg, E. (1996). La planificación educativa ( $7^{\mathrm{a}}$. ed.). Argentina: Editorial Mc-Graw Hill.

Bueno, E. (2003). Organización de empresas. Estructura, proceso y modelos. Madrid, España: Ediciones Pirámide, S. A.

Castellano, B. (2001). El oficio del planificador. Caracas, Venezuela: Editores Hermanos Vadell.

Chávez, N. (2004). Introducción a la Investigación Educativa. Maracaibo, Venezuela: Editorial Universo.

Gibson, I., y Donnely, J. (2001). Las organizaciones ( $8^{\mathrm{a}}$. ed.). Colombia: Editorial Mc-Graw Hill Interamericana, S.A.

Gómez, C. (2003). Componentes de la administración. Caracas, Venezuela: Universidad Central de Venezuela.

Hernández, R., Fernández, C., y Batista, P. (2006). Metodología de la investigación. México: Editorial Mc GrawHill.

Kaufman, R. (2006). Planificación de sistemaseducativos. México: Editorial Trillas. México.

Koontz, H. y Heinz, W. (2002). Administración: Una perspectiva global (10 ${ }^{\mathrm{a}}$. ed.). México: Editorial Mc Graw Hill.

Melinkoff, K. (2004). Los procesos administrativos. Caracas,Venezuela, Editorial Panamá.

Molina, M. (2001). Introducción a la planificación general. Caracas, Venezuela: Editorial Cornel. 
Prieto, E. (2002). La planificación de actividades en el aula como estrategia para mejorar el rendimiento estudiantil en matemática. (Tesis de postgrado sin publicar). Universidad del Zulia. Maracaibo, Venezuela.

Robbins, S. (2001). Administración: Teoría y práctica (4 ${ }^{\mathrm{a}}$. ed.). México: Editorial Prentice Hall Hispanoamericana, S. A.

Rosales, P. (2002). Planificación de la enseñanza. Mérida, Venezuela: Editorial Universidad de los Andes.
Shein, E. (2004). La cultura empresarial y el liderazgo. Barcelona: Editorial Plaza \& Jones.

Smith, C. (2002). Guías para supervisores. México: Editorial Trillas.

Stoner, J., Freeman, E y Gilbert, D. (2004). Administración (5 ${ }^{\mathrm{a}}$. ed.). México: Prentice Hall Hispanoamericana, S.A.

Venezuela. Ministerio de Educación. (2005). ¿Cómo entendemos, elaboramos y ejecutamos los Proyectos Pedagógicos de Plantel (P.P.P.)? Caracas, Material mimeografiado. 
